\title{
Performance of Diversity Combining ARQ Error Control in a TDMA SC-FDE System
}

\author{
Miguel Pereira, Student Member, IEEE, Luis Bernardo, Member, IEEE, Rodolfo Oliveira, Member, IEEE, \\ Paulo Carvalho, and Paulo Pinto, Member, IEEE
}

\begin{abstract}
The Quality of Service (QoS) requirements for modern broadband wireless systems can be very high, with small error rates and delays. Packets with errors are usually discarded and need to be retransmitted, leading to performance degradation. An alternative to simple retransmissions like Automatic Repeat reQuest (ARQ) schemes, that can minimize the degradation is to combine the signals associated to different transmission attempts, usually called diversity combining techniques.

This paper considers the use of Single-Carrier (SC) modulations with Frequency-Domain Equalization (FDE) and lowcomplexity soft combining ARQ schemes. The time diversity combining technique presented in this paper, allows packets associated to different transmission attempts to be combined in a soft way so as to improve the performance. This technique is employed in a Time Division Multiple Access (TDMA) scheme. An accurate analytical model is proposed for the evaluation of the uplink packet delay in non-saturated traffic condition, and for the system's throughput (goodput) considering a generic packet arrival process. Physical (PHY) layer (packet error rates) and system-level (goodput and packet delay considering both PHY and Medium Access Control (MAC) layers) results are presented and validated by simulations for two distinctive arrival processes: Poisson and Geometric.
\end{abstract}

Index Terms-Hybrid-ARQ techniques, MAC protocol, TDMA schemes, queuing theory, generic packet arrival distribution, Markov chains.

\section{INTRODUCTION}

$\mathbf{P}$ ACKET errors can be significant in wireless systems due to fading and shadowing effects, which may lead to significant decrease in the receiving power. The traditional approach to cope with an erroneous packet is to discard it, and ask for its retransmission, which corresponds to the conventional Automatic Repeat reQuest techniques (ARQ). The major problem with conventional ARQ techniques is that persistent unfavorable propagation conditions may originate a very high Packet Error Rate (PER). The individual packet error probability is not affected by the number of packet retransmissions because the information contained in the signal of the discarded packets is not used. Hybrid ARQ/Forward

Paper approved by V. A. Aalo, the Editor for Diversity and Fading Channel Theory of the IEEE Communications Society. Manuscript received February 9, 2011; revised July 25, 2011.

A preliminary and reduced version of this paper was presented at IEEE WCNC'10.

This work was supported by FCT/MEC (CTS multi-annual funding project PEst-OE/EEI/UI0066/2011, IT pluri-annual funding project PEst-OE/EEI/LA0008/2011, MPSat project PTDC/EEA-TEL/099074/2008, OPPORTUNISTIC-CR project PTDC/EEA-TEL/115981/2009, and grant SFRH/BD/41515/2007)

The authors are with the FCT-UNL - Universidade Nova de Lisboa, Instituto de Telecomunicações and UNINOVA, Portugal (e-mail: miguelpereira.pro@gmail.com; \{lflb, rdinis, rado,pmc,pfp\}@fct.unl.pt).

Digital Object Identifier 10.1109/TCOMM.2012.013112.110096
Error Correction (FEC) strategies were proposed [1]-[7] to cope with these scenarios. This paper considers only the TypeII strategies, which retain the signal associated to an erroneous packet and that may ask for additional redundancy. These packet-combining (PC) systems can be loosely arranged into two categories [8]: code-combining systems and diversitycombining systems. In code-combining (CC) systems [1]-[5], the packets are concatenated to form noise-corrupted code words from increasingly longer and lower rate codes. The CC approach relies on a powerful error correcting code that is punctured, to increase the data rate. Successive retransmissions reduce the puncturing, and therefore, increase the error correction capacities of the code. This limits the error correction capability to the base code capabilities.

In diversity-combining (DC) systems [6], [7], the individual symbols from multiple, identical copies of a packet are combined to create a single packet with more reliable constituent symbols. They are based on repetition codes with a soft decision, which are not bounded by the performance of the basic code. DC systems are simpler to implement, and allow efficient implementations with performances comparable to the CC systems when iterative detectors are used [7].

The use of Hybrid-ARQ (H-ARQ) techniques improves the network throughput compared to conventional ARQ techniques because the packet error probability $\left(p_{i}\right)$ for the retransmission $i$ is usually lower than $p_{i-1}$. One of the purposes of this paper is to study the performance of a more comprehensive system than the current research approaches. We consider a Time Division Multiple Access (TDMA) scheme, where a station shares a multiple access communication channel by transmitting its packets during its dedicated time slots, and a generic packet arrival distribution function. We can classify the current research in two main areas: one that approaches the problem focusing mainly on the transmission techniques to lower the packet error rate and consequent delay and throughput; another that focuses on studying the delay based on queueing theory and the usage of TDMA.

The first group uses analytical models or Monte Carlo simulation studies (e.g. [9]). Kallel [10] analyzed the maximum throughput for the [2] H-ARQ scheme, using convolutional coding and exploiting code combining. Zhang et al. [11], [12] proposed a selective combining approach, and analyzed its transmission delay and throughput for a Rayleigh fading channel. Fading channel support was further extended, considering: noisy feedback and truncated ARQ [13]; information-theoretic performance for random access with random Gaussian codes [14]; the performance of low-density parity-check (LDPC) code ensembles with iterative belief-propagation decoding 
[15]; and an integrated approach that adds the static channel information to the sum of Signal-to-Noise Ratio (SNR) [16]. Huang et al. [17] analyses the optimal scheduling policy for an H-ARQ downlink, which maximizes the link's throughput (minimizes the number of retransmissions) for Poisson arrival processes. Boujemâa et al. [18] analyzed the maximum throughput performance of H-ARQ schemes with diversity and code combining using Direct Sequence Code Division Multiple Access (DS-CDMA) with a Rake receiver. They also analyzed [19] the delay of cooperative truncated H-ARQ with opportunistic relaying for Poisson sources, where relay nodes with better SNR may handle retransmissions. Choi et al. [20] and Le Duc et al. [21], [22] considered the HARQ performance for the group of fragments that compose an Internet Protocol (IP) packet. They proposed IP-Medium Access Control (MAC) cross-layer optimizations and a model for the IP packet error rate and transmission delay. Luo et al. [23] analyzed the Service Data Unit (SDU) delivery delay of selective-repeat ARQ (SR-ARQ) as a function of the SDU size and the channel coding scheme. Badia et al. [24], [25] analyzed the SR-ARQ packet delay statistics using Markov channel models and assuming a constant round-trip time, which originate periodic packet retransmissions. Paper [25] extends [24] (uncoded systems), analyzing the performance of SR-ARQ with a truncated type II H-ARQ technique based on Reed Solomon erasure codes. Bauch [26] and Gore [27] introduced the concept cyclic delay diversity in the channel frequency with the objective to mitigate channel correlations across multiple retransmissions. This concept was also applied on H-ARQ schemes by Gidlund [28].

TDMA is used in several current wireless network systems (e.g. 802.16[29], LTE[30], etc.) when hard QoS (Quality of Service) guarantees are needed. TDMA was the focus of an extensive list of past works (e.g. [31]-[36]), which assumed a constant packet error probability for the different retransmissions. Lam [31] considered an errorless TDMA system for Poisson traffic, and Khan [33] relaxed the traffic assumptions. ARQ error control was analyzed by Saeki and Rubin [32] for a Poisson distributed packet arrival process. Rubin and Zhang [34] analyzed the packet delay for TDMA using multiple contiguous-slot assignments and considering a generic packet arrival process and a geometric packet delay. This model can be used for a conventional ARQ approach when the transmission and processing delays are not taken into account. Neuts et al. [35] considered a finite buffer TDMA system with Poisson traffic. Chen and Chang [36] proposed a delay analysis model for TDMA where the slot assignment is characterized by a random variable, for a Poisson distributed packet arrival process. Seo et al. [37] presented a study for an Hybrid-ARQ scheme, considering a M/G/1 model with convolutional codes, when a maximum of two packets are combined.

In this paper, we study the performance of H-ARQ techniques for SC-FDE (Single Carrier with Frequency-Domain Equalization) schemes. SC-FDE schemes are generally accepted as one of the best candidates for the uplink of future broadband wireless systems [38]. Particularly, we propose and analyze a new H-ARQ retransmission strategy for SC-FDE where the transmitter employs a shifted packet approach to

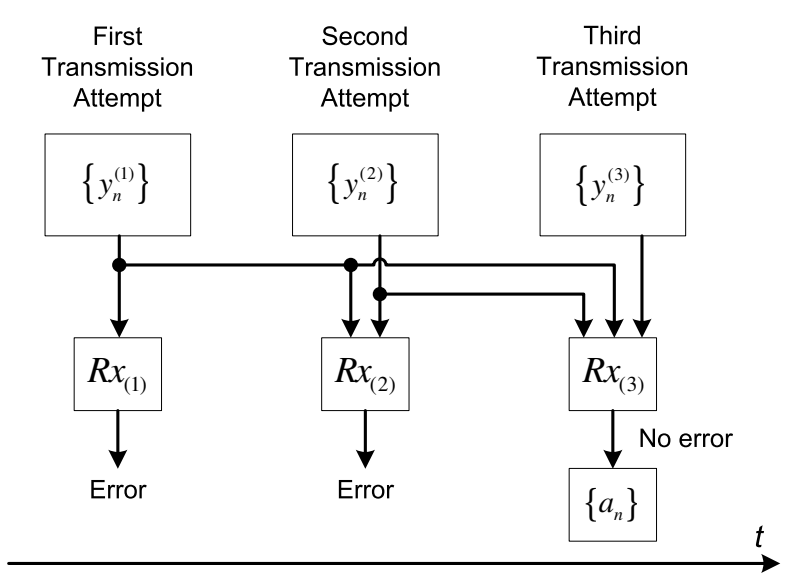

Fig. 1. Receiver process.

improve the H-ARQ performance particularly suited when the channel presents severely time-dispersive characteristics. Considering a TDMA scheme, we describe a new analytical model valid for a generic H-ARQ TDMA scheme. To the best of our knowledge, this paper is the first to propose an accurate model concerning the queue-size and the packet delay analysis of a TDMA system with an H-ARQ Type-II technique, for a generic packet arrival distribution. Numerical simulation results are presented in order to validate the system characterization. The analytical model is also validated using ns2 [39] simulations, showing its accuracy. Results concerning goodput and packet delay using two traffic distributions (Poisson and Geometric) and taking into account the transmission technique simulations, are properly addressed. As expected, H-ARQ reduces significantly its packet delay compared to the conventional ARQ technique.

Although our analytical model can be applied to any transmission technique, we considered its application in SC-FDE schemes. This model can be viewed as a tool for system analysis and for system configuration (e.g. determine the minimum $E_{b} / N_{0}$ value that satisfies an average delay bound given a traffic model bound).

The paper is organized as follows: the system overview, including our soft packet combining technique is presented in Section II. The system performance is evaluated in Section III. Performance results are presented in Section IV. Finally, Section V is concerned with the conclusions of this paper.

\section{H-ARQ SYSTEM DESIGN}

In this paper, we consider the uplink transmission on a SC-FDE system. As with other FDE systems, data is transmitted in fixed size blocks and the time-domain block associated to a given user (i.e., the corresponding packet) is $\left\{s_{n} ; n=0,1, \ldots, N-1\right\}$, where $s_{n}$ is a data symbol selected from a given constellation. To each time-domain block $\left\{s_{n} ; n=0,1, \ldots, N-1\right\}$ we associate a frequencydomain block $\left\{S_{k} ; k=0,1, \ldots, N-1\right\}$ which is the Discrete Fourier Transform (DFT) of $\left\{s_{n} ; n=0,1, \ldots, N-1\right\}$. As with other block transmission techniques, a suitable cyclic prefix is added to each time-domain block. 
When packet errors are detected we ask for its retransmission, but the signal associated to each transmission attempt is stored as shown in Fig. 1. The packet associated to the $r$ th attempt to transmit $\left\{s_{n} ; n=0,1, \ldots, N-1\right\}$ is $\left\{s_{n}^{(r)} ; n=0,1, \ldots, N-1\right\}$.

\section{A. Receiver Characterization}

Let us assume that we have $l$ versions of the packet (i.e., there were $l$ transmission attempts ${ }^{1}$ ) and our receiver combines them in an efficient way. The received signal associated to the $r$ th transmission attempt is sampled and the cyclic prefix is removed, leading to the time-domain block $\left\{y_{n}^{(r)} ; n=\right.$ $0,1, \ldots, N-1\}$. If the cyclic prefix is longer than the overall channel impulse response then the corresponding frequencydomain block is $\left\{Y_{k}^{(r)} ; k=0,1, \ldots, N-1\right\}$, where

$$
Y_{k}^{(r)}=S_{k}^{(r)} H_{k}^{(r)}+N_{k}^{(r)}, \quad r=1,2, \ldots, l,
$$

with $N_{k}^{(r)}$ denoting the channel noise. $H_{k}^{(r)}$ is the overall channel frequency response for the $r$ th transmission attempt and $\left\{S_{k}^{(r)} ; k=0,1, \ldots, N-1\right\}$ is the DFT of $\left\{s_{n}^{(r)} ; n=\right.$ $0,1, \ldots, N-1\}$.

Our receiver, which is based on the Iterative Block-Decision Feedback Equaliser (IB-DFE) receivers proposed in [40], is depicted in Fig. 2. We have an iterative frequency-domain receiver where, for a given iteration $i$, the frequency-domain samples at the output are given by

$$
\tilde{S}_{k}^{(i)}=\sum_{r=1}^{l} F_{k}^{(r, i)} Y_{k}^{(r)}-B_{k}^{(i)} \bar{S}_{k}^{(i-1)}
$$

where $\left\{F_{k}^{(r, i)} ; k=0,1, \ldots, N-1\right\}(r=1,2, \ldots, l)$ and $\left\{B_{k}^{(i)} ; k=0,1, \ldots, N-1\right\}$ can be regarded as the feedforward and the feedback coefficients, respectively. $\left\{\bar{S}_{k}^{(i-1)} ; k=\right.$ $0,1, \ldots, N-1\}$ denotes the DFT of the average data estimates $\left\{\bar{s}_{n}^{(i-1)} ; n=0,1, \ldots, N-1\right\}$, where $\bar{s}_{n}$ denotes the average symbol values conditioned to the FDE output. For Quadrature Phase Shift Keying (QPSK) constellations these average values are given by

$$
\bar{s}_{n}^{(i)}=\tanh \left(\frac{L_{n}^{I(i)}}{2}\right)+j \tanh \left(\frac{L_{n}^{Q(i)}}{2}\right)
$$

(without loss of generality, we assume that $\left|s_{n}\right|^{2}=2$, i.e., $s_{n}= \pm 1 \pm j$ ), with $L_{n}^{I(i)}=\frac{2}{\sigma_{e q}^{2}} \operatorname{Re}\left\{\tilde{s}_{n}^{(i)}\right\}$ and $L_{n}^{Q(i)}=$ $\frac{2}{\sigma_{e q}^{2}} \operatorname{Im}\left\{\tilde{s}_{n}^{(i)}\right\}$ denoting the LogLikelihood Ratios (LLRs) of the "in-phase bit" and the "quadrature bit", associated to $s_{n}$, respectively, and $\left\{\tilde{s}_{n}^{(i)} ; n=0,1, \ldots, N-1\right\}=$ Inverse DFT $\left\{\tilde{S}_{k}^{(i)} ; k=0,1, \ldots, N-1\right\}$. The variance $\sigma_{e q}^{2}$ is given by

$$
\sigma_{e q}^{2}=\frac{1}{2} E\left[\left|s_{n}-\tilde{s}_{n}^{(i)}\right|^{2}\right] \approx \frac{1}{2 N} \sum_{n=0}^{N-1}\left|\hat{s}_{n}^{(i)}-\tilde{s}_{n}^{(i)}\right|^{2},
$$

where $\hat{s}_{n}^{(i)}= \pm 1 \pm j$ are the hard-decisions associated to $\tilde{s}_{n}^{(i)}$.

$$
{ }^{1} r=1,2, \ldots, l
$$

The optimum feedforward coefficients for a given iteration, can be written as (see [7])

$$
F_{k}^{(r, i)}=\frac{\mathcal{K}^{(i)} H_{k}^{(r) *}}{\alpha+\left(1-\left(\rho^{(i-1)}\right)^{2}\right) \sum_{r^{\prime}=1}^{l}\left|H_{k}^{\left(r^{\prime}\right)}\right|^{2}}, r=1,2, \ldots, l,
$$

where

$$
\alpha=\frac{E\left[\left|N_{k}^{(r)}\right|^{2}\right]}{E\left[\left|S_{k}\right|^{2}\right]}
$$

(i.e., $\alpha$ is the inverse of the SNR), the correlation coefficient $\rho^{(i)}$ is given by

$$
\rho^{(i)}=\frac{1}{2 N} \sum_{n=0}^{N-1}\left(\left|\operatorname{Re}\left\{\bar{s}_{n}^{(i)}\right\}\right|+\left|\operatorname{Im}\left\{\bar{s}_{n}^{(i)}\right\}\right|\right)
$$

and $\mathcal{K}^{(i)}$ is selected so that $\frac{1}{N} \sum_{k=0}^{N-1} \sum_{r=1}^{l} F_{k}^{(r, i)} H_{k}^{(r)}=1$.

The optimum feedback coefficients (also to minimize the signal-to-noise plus interference ratio) are given by (see [7])

$$
B_{k}^{(i)}=\sum_{r=1}^{l} F_{k}^{(r, i)} H_{k}^{(r)}-1 .
$$

For the first iteration $(i=1)$ we do not have any information about $S_{k}$ and the correlation coefficient $\rho^{(0)}$ is zero. This means that $B_{k}^{(1)}=0$ and

$$
F_{k}^{(r, 1)}=\frac{H_{k}^{(r) *}}{\alpha+\sum_{r^{\prime}=1}^{l}\left|H_{k}^{\left(r^{\prime}\right)}\right|^{2}}, \quad r=1,2, \ldots, l,
$$

corresponding to the optimum coefficients for a linear FDE [41].

The feedforward coefficients, can be written as

$$
F_{k}^{(r, i)}=H_{k}^{(r) *} C_{k}^{(i)}, r=1,2, \ldots, l
$$

with

$$
C_{k}^{(i)}=\frac{1 / \mathcal{K}^{(i)}}{\alpha+\left(1-\left(\rho^{(i-1)}\right)^{2}\right) \sum_{r^{\prime}=1}^{l}\left|H_{k}^{\left(r^{\prime}\right)}\right|^{2}} .
$$

This means that the bank of feedforward filters can be replaced by a bank of matched filters, corresponding to an ideal Maximum Ratio Combining (MRC), followed by a single feedforward filter characterized by the set of coefficients $\left\{C_{k}^{(i)} ; k=0,1, \ldots, N-1\right\}$.

\section{B. Coping With Static Channel Conditions}

In conventional ARQ systems when the transmitter receives a retransmission request (e.g., a Negative Acknowledgement (NACK) or a suitable timeout), it simply retransmits the erroneous packet. Ideally, it would be better to have different channel conditions not to repeat the failure, i.e., the channel conditions should be uncorrelated from retransmission to retransmission attempt. We denote this condition as Uncorrelated Channel (UC). Typically, to have UC conditions we need to change the working band for each retransmission attempt (something not practical), and/or we need to have an interval between retransmissions large enough to allow significant channel variations due to Doppler effects (which might lead to significant delays in the ARQ case), and/or the 